\title{
¿Qué hacer cuando el paciente es un colega? Dos años de experiencia del programa de Atención Integral al Médico Enfermo (PAIME)
}

\author{
Arteman. A.; Gual, A.; Padrós, J.; Casas, M.; Bruguera, E.; Colom, J. \\ Programa d'Atenció Integral al Metge Malalt.
}

Enviar correspondencia a:

PAIMM. Colegio Oficial de Médicos de Barcelona. Paseo Bonanova, 47. 08017 BARCELONA. Tel. 9356788 56. E-mail: paimm@comb.es

\begin{abstract}
RESUMEN
Este trabajo expone las razones que han motivado la creación del programa PAIMM por parte de los Colegios de Médicos de Cataluña y del Departamento de Sanidad y Seguridad Social de la Generalitat. Los problemas de los profesionales de la sanidad y sobre todo de los médicos, que padecen trastornos psíquicos y adictivos para acudir al sistema sanitario a recibir el tratamiento adecuado, son varios y diversos, pero la falta de confidencialidad es el más importante. Se describen también las características del programa y de su Unidad de Tratamiento, con los últimos datos asistenciales.
\end{abstract}

Palabras clave: médicos enfermos, trastornos mentales y adictivos, confidencialidad, demanda voluntaria, rehabilitación.

\section{ABSTRACT:}

The target of this paper is to explain the reasons of PAIMM programme creation by Medical Associations of Catalonia and Health Department of Generalitat, (Autonomous Government). Problems of health professionals, physicians above all, who suffer mental and addictive disorders are several and diverse, but the lack of confidentiality is the most important. We also describe the headlines of the Programme and its Treatment Unit, and we expose last figures too.

Key words: sick physicians, mental and addictive disorders, confidentiality, voluntary demand, rehabilitation.
$\mathbf{P}$ or lo general, cuando los profesionales de la sanidad padecen algún problema de salud no suelen pedir ayuda (1), o en todo caso, está demostrado que lo hacen en mucha menor proporción que la población general. (2) Además, cuando el problema de salud es un trastorno mental, con o sin adicción al alcohol o a otras sustancias incluidas los psicofármacos, entonces prácticamente nunca piden ayuda. (3) En el caso de los médicos, esta reacción parece ser todavía más acusada que en el resto de profesiones sanitarias.

El miedo a ser identificados como enfermos mentales o drogadictos, con todo el estigma de estas enfermedades, que todavía perdura incluso entre la profesión, la vergüenza por no haber sido capaces de controlarse y de superar la enfermedad, el miedo a perder el trabajo e incluso a no poder ejercer más, son factores que pesan más que el sentido común en estas situaciones. (4) Situaciones que hacen que estos médicos enfermos no pidan ayuda y por lo tanto, continúen trabajando en sus centros, tomando decisiones clínicas día a día, con mayores probabilida- des de cometer errores y negligencias, y por tanto, con mayor riesgo para la salud de sus pacientes. (5)

Los sistemas de salud europeos no tienen prevista ninguna actuación especial para prevenir y atender estos casos, y en España, hasta ahora, no somos una excepción. Nuestras instituciones sanitarias, sean de un Servicio Autonómico de Salud con servicios transferidos, sean del propio INSALUD, no disponen de ningún programa o dispositivo asistencial para atender estos casos, fuera de los usuales para la población general. Y lo que suele suceder en estos casos, es que no se haga ninguna intervención institucional adecuada, que estos enfermos no pidan ayuda y no acudan a los dispositivos asistenciales normales. El problema pues, se vuelve crónico con las consiguientes repercusiones graves para la convivencia familiar, para el rendimiento profesional y para la calidad de la asistencia en general. Finalmente, se ponen en marcha los únicos mecanismos existentes para estas situaciones límite: los expedientes disciplinarios y las propuestas de invalidez laboral. 
Como puede apreciarse, las características que presentan tales situaciones son muy peculiares $y$, si se quieren prevenir y resolver, exigen una forma de abordar y atender los casos muy determinada, que por lo general los dispositivos asistenciales normales no pueden ofrecer.

En este sentido, hay que destacar que el factor que pesa más y que condiciona todos los demás es la confidencialidad. Es del todo necesario, imprescindible diríamos, que para que los médicos enfermos tengan confianza y acudan a ser tratados, el circuito de acceso a la atención y los propios servicios asistenciales deben ser confidenciales. Cuando ello no es así, los médicos enfermos simplemente no acuden, no son tratados y siguen trabajando, que es lo que hasta ahora está sucediendo. Esto nos lleva a que el dispositivo asistencial deba ser específico para estos casos, sino no puede ser confidencial, lo uno conlleva lo otro.

Otro de los factores importantes para abordar con éxito este problema es que dicho dispositivo asistencial específico disponga de terapeutas especializados en estas enfermedades y en tratar a médicos enfermos, ya que éstos suelen ser pacientes difíciles, acostumbrados a hacer de médicos terapeutas pero no de médicos enfermos y por tanto pacientes, poco cumplidores, a veces con escasa conciencia de enfermedad, pero siempre con extensos conocimientos de medicina y probablemente de su propia enfermedad.

Conscientes de esta problemática, los Colegios de Médicos de Cataluña conjuntamente con el Departamento de Sanidad de la Generalitat, acordaron a finales de 1998 crear el Programa de Atención Integral al Médico Enfermo (PAIME) (6), un programa específico para atender a los médicos que padecían algún trastorno psíquico y/o alguna adicción. La finalidad del PAIME es pues, la de atender a estos médicos enfermos, para recuperarlos para el ejercicio de la profesión, asegurando al mismo tiempo que la calidad de dicho ejercicio sea la mayor posible.

EI PAIME está gestionado por el Colegio Oficial de Médicos de Barcelona, se financia por la Generalitat mayormente y por los cuatro colegios de médicos de Cataluña, se rige por un procedimiento colegial único para el acceso y acogida de los casos, y funciona bajo criterios de estricta confidencialidad. Sus servicios son gratuitos para los médicos colegiados de Cataluña y se espera que en el futuro se establezcan acuerdos con otras comunidades autónomas y colegios de médicos de España para extender esta gratuidad a sus profesionales.

EI PAIME dispone de una Unidad de Tratamiento propia, específica y especializada, con áreas separadas de servicios ambulatorios y de internamiento. Desde su creación se han atendido a un total de 204 casos de médicos, principalmente de Cataluña, pero también del resto de España (un $12 \%$.) (7) En estos últimos casos, se trata de actuar en estrecha coordinación con el terapeuta que deriva el caso y si no lo hay, es decir cuando la demanda ha sido personal y espontánea, se procura, siempre con el consentimiento del médico enfermo, derivarle a un especialista de su territorio en el momento del alta del internamiento, para su seguimiento ambulatorio.

Es importante señalar que debido a su especificidad, especialización y tamaño forzosamente reducido, la unidad de internamiento del PAIME tiene unos costes económicos muy elevados. Por lo que no parece razonable, al menos desde el punto de vista económico y de rendimiento asistencial, la creación de otras unidades de internamiento en el territorio español. En cambio, seria muy recomendable la puesta en marcha de programas colegiales similares al PAIME con unidades de tratamiento ambulatorio en todas las comunidades autónomas.

El $81 \%$ de los casos atendidos ha accedido al PAIME por demanda voluntaria, lo que nos indica el alto grado de confianza obtenido entre la profesión. El acceso al programa mediante comunicación confidencial de un familiar, colega o superior, ha sido del $13 \%$ y siempre antes, se ha procurado reconvertir el acceso a demanda voluntaria del propio afectado, gestionando el caso con su entorno familiar y/o laboral. (7)

Los motivos de demanda han sido en primer lugar los trastornos psíquicos con un $51 \%$, seguido de la adicción al alcohol en un $31 \%$ y al resto de drogas en un $18 \%$, aunque en una gran parte de los casos se presenta patología dual, psiquiátrica y adictiva. (7)

Las edades se concentran mayoritariamente entre los 35 y 55 años, pero si se calcula la tasa de incidencia por grupo de edad se observa que están muy repartidas en cada grupo etario. En cuanto al género, predominan los hombres en una proporción de 3,5 a 1. (7)

La importancia del PAIME como experiencia pionera en Europa ha sido destacada en revistas médicas de ámbito internacional. (8)(9)

Desde hace unos meses el Colegio de Diplomados de Enfermería de Barcelona, ha puesto en marcha un programa similar al PAIME denominado RETORNO, que utiliza la misma Unidad de Tratamiento y que se está extendiendo al resto de colegios de enfermería de Cataluña. (10)

\section{BIBLIOGRAFÍA}

(1) Bruguera M. y otros. La actitud de los médicos en relación a su propia salud. Art. en preparación. Feb 2001.

(2) Angers D., Talbott, G.D.; Healing the healers. The addicted physician. Libro. Edi. Psychosocial Press. 1998. 
(3) Walzer RS. Impaired physicians: an overview and update of the legal issues. J Legal Med 1990; 11: 131-198.

(4) O'Connor PG, Spickard A. Physician impairment by substance abuse. Med Clin N Amer 1997; 81:1037-1052.

(5) Centrella M. Physician addiction and impairment-current thinking: a review. J Addictive Dis 1994;13: 91-105.

(6) Convenio de Colaboración entre el Servicio Catalan de la Salud y el Departamento de Sanidad y Seguridad Social de la Generalitat de Cataluña, por una parte, y por otra, el Colegio Oficial de Médicos de Barcelona, para la creación y puesta en funcionamiento del Programa de Atención Integral al Médico Enfermo (PAIME). Barcelona, 15-09-1998.
(7) Memoria del PAIMM, 1998-2000. Colegio Oficial de Médicos de Barcelona. En prensa. Barcelona. Feb 2001.

(8) The Lancet. 1998; 352; 9133: 999 y 1045.

(9) JAMA. 2000; 283; 24: 3186.

(10) Cláusua de modificación del convenio de colaboración subscrito entre el Servicio Catalan de la Salud y el Departamento de Sanidad y Seguridad Social de la Generalitat de Cataluña, por una parte, y por otra, el Colegio Oficial de Médicos de Barcelona, para la creación y puesta en funcionamiento del PAIME para extender su actuación a los profesionales de enfermeria. Barcelona, 28-12-2000. 
